Freiburg-THEP 97-08

March 1997

\title{
Radiative corrections to the lineshape of a heavy Higgs boson at muon colliders
}

\author{
Thomas Binoth and Adrian Ghinculov \\ Albert-Ludwigs-Universität Freiburg, Fakultät für Physik \\ Hermann-Herder Str.3, D-79104 Freiburg, Germany
}

\begin{abstract}
We study the NNLO radiative corrections of enhanced electroweak strength to the lineshape of the Higgs boson in the processes $\mu^{+} \mu^{-} \rightarrow H \rightarrow Z Z$ and $\mu^{+} \mu^{-} \rightarrow H \rightarrow t \bar{t}$, which are of interest for Higgs searches at a possible muon collider. We give the radiative corrections both in the on-shell renormalization scheme and in the pole renormalization scheme. The pole scheme appears to have better convergence properties, in agreement with previous results regarding the position of the Higgs pole in the complex plane.
\end{abstract}




\title{
Radiative corrections to the lineshape of a heavy Higgs boson at muon colliders
}

\author{
Thomas Binoth and Adrian Ghinculov \\ Albert-Ludwigs-Universität Freiburg, Fakultät für Physik, \\ Hermann-Herder Str.3, D-79104 Freiburg, Germany
}

\begin{abstract}
We study the NNLO radiative corrections of enhanced electroweak strength to the lineshape of the Higgs boson in the processes $\mu^{+} \mu^{-} \rightarrow H \rightarrow Z Z$ and $\mu^{+} \mu^{-} \rightarrow H \rightarrow t \bar{t}$, which are of interest for Higgs searches at a possible muon collider. We give the radiative corrections both in the on-shell renormalization scheme and in the pole renormalization scheme. The pole scheme appears to have better convergence properties, in agreement with previous results regarding the position of the Higgs pole in the complex plane.
\end{abstract}

As higher loop calculation are becoming available in the electroweak symmetry breaking sector of the standard model, one is able to perform phenomenological studies of processes relevant for Higgs searches at future colliders at higher orders in perturbation theory. The precision of such studies is restricted by the renormalization scheme dependence which enters through the truncation of the perturbative series of a given observable.

The scheme dependence is formally one order higher than the order calculated. This is a small effect as long as the the coupling constant is small. As the coupling constant increases, the sizes of different loop order corrections are growing, the scheme dependence of the predictions increases, and the perturbative result is affected by large theoretical uncertainties. By comparing several schemes with respect to the size of the loop corrections, one can get some insight on the reliabilty of the perturbative calculation in these schemes. One is especially interested in schemes which have better convergence properties. Because the mass of the Higgs boson is related to the quartic coupling of the Higgs field, such effects must be taken into account when performing phenomenological studies related to the search for a heavy Higgs boson.

In ref. [四] we studied the position of the pole of the Higgs propagator at three-loop level. There we concluded that it appears to be more convenient to describe heavy Higgs bosons in terms of the pole renormalization scheme instead of the widely used on-shell scheme. We suggested that this may be the result of the presence in the 
Higgs sector of a nonperturbative mechanism similar to the effect discussed in ref. [2] for the case of a simpler model which admits a solution at all orders.

In this letter we study the NNLO radiative corrections for the reactions $\mu^{+} \mu^{-} \rightarrow$ $H \rightarrow Z Z$ and $\mu^{+} \mu^{-} \rightarrow H \rightarrow, t \bar{t}$. These processes were already studied at leading order as a source of Higgs bosons at a possible muon collider [3]. We give the radiative corrections both in the on-shell and the pole renormalization schemes, and compare the two schemes. The results support the previous conclusion about the convergence of perturbative expansions in the pole scheme versus the on-shell scheme.

Let us start with the on-shell scheme, in which most of the existing one- and two-loop results were derived. In the following we will calculate the ratios between the amplitudes of the processes considered, including the radiative corrections up to the desired order, and the corresponding amplitudes in the Born approximation:

$$
\begin{aligned}
K_{1}(s) & =V_{H \mu^{+} \mu^{-}} \frac{s-m_{H}^{2}+i m_{H} \Gamma_{\text {tree }}}{s-m_{H}^{2}+i m_{H} \Pi(s)} V_{H t \bar{t}} \\
K_{2}(s) & =V_{H \mu^{+} \mu^{-}} \frac{s-m_{H}^{2}+i m_{H} \Gamma_{\text {tree }}}{s-m_{H}^{2}+i m_{H} \Pi(s)} V_{H Z Z}(s)
\end{aligned}
$$

These factors can easily be included in a Monte Carlo simulation for taking into account the NNLO radiative corrections.

In the equations above, $\Pi(s)$ is the Higgs self-energy, and in the resonance region plays essentially the role of an $s$ dependent width. $V_{H \mu^{+} \mu^{-}} \equiv V_{H t \bar{t}}$ and $V_{H Z Z}(s)$ are the radiative correction factors to the Yukawa couplings, and to the Higgs coupling to the weak vector bosons, respectively. $\Pi$ and $V_{H Z Z}$ are functions of $s$, while the corrections to the Yukawa couplings are momentum-independent at leading order in the Higgs mass [9, 10].

The existing one- and two-loop results in the Higgs sector allow one to determine the correction factors $K_{1}$ and $K_{2}$ at NNLO. The vertex corrections are known up to $\mathcal{O}\left(\lambda^{2}\right)$, and the Higgs selfenergy is known to $\mathcal{O}\left(\lambda^{3}\right)$. In the resonance region the instability of the Higgs boson needs to be taken into acoount by means of a Dyson summation. This introduces inverse powers of the coupling $\lambda$. At the same time, in order to keep all contributions relevant at the order considered, one must remember that in the resonance region the quantity $\delta=\left(s-m_{H}^{2}\right) / m_{H}^{2}$ is of order $\lambda$. Therefore one has to perform a double expansion in $\delta$ and $\lambda$ of the quantities entering the expressions of $K_{1}$ and $K_{2}$ [1, 11]:

$$
\begin{aligned}
\Pi(s) / m_{H} & =A+\delta B+\delta^{2} C+\mathcal{O}\left(\delta^{3} \lambda\right) \\
A & =a_{1} \lambda+a_{2} \lambda^{2}+a_{3} \lambda^{3}+\mathcal{O}\left(\lambda^{4}\right) \\
B & =b_{1} \lambda+b_{2} \lambda^{2}+\mathcal{O}\left(\lambda^{3}\right)
\end{aligned}
$$




$$
\begin{aligned}
C & =-i c_{1} \lambda+\mathcal{O}\left(\lambda^{2}\right) \\
V_{H \mu \mu, H t \bar{t}} & =1+d_{1} \lambda+d_{2} \lambda^{2}+\mathcal{O}\left(\lambda^{3}\right) \\
V_{H z z}(s) & =1+e_{1} \lambda+e_{2} \lambda^{2}+f_{1} \delta \lambda+\mathcal{O}\left(\lambda^{3}\right)
\end{aligned}
$$

The necessary coefficients $a_{i}-f_{i}$ can be extracted from one- and two-loop calculations already available [5]-13]:

$$
\begin{aligned}
& a_{1}=3 \pi / 8, \quad a_{2}=a_{1} \cdot 0.350119, \quad a_{3}=a_{1} \cdot(0.97103+0.000476), \\
& b_{1}=0, \quad b_{2}=1.002245, \quad c_{1}=0.2181005, \\
& d_{1}=0.132325, \quad d_{2}=-0.26387, \\
& e_{1}=0.17505951-i 1.4190989, \quad e_{2}=-0.53673-i 0.32811, \\
& f_{1}=0.42536605-i 0.15169744 .
\end{aligned}
$$

These constants define the correction factors $K_{1}$ and $K_{2}$ around the Higgs resonance correct at NNLO in the on-shell renormalization scheme (OS). In this scheme, the Higgs mass $m_{H}$ is defined by the zero of the real part of the inverse Higgs propagator. In the pole scheme (PS) the mass $M$ of the Higgs boson is defined by the real part of the location of the pole in the complex $s$ plane.

To relate to the pole renormalization scheme, one needs the relation between the on-shell mass $m_{H}$ and the pole mass $M$. Such a relation, correct at order $\lambda^{3}$, was derived in refs. [6, 1]:

$$
M^{2}=m_{H}^{2}\left[1+\frac{a_{1}^{2}}{4}\left(\frac{G_{F}}{\sqrt{8} \pi^{2}}\right)^{2} m_{H}^{4}+\frac{a_{1} a_{2}-2 a_{1} b_{2}+2 a_{1}^{2} c_{1}}{2}\left(\frac{G_{F}}{\sqrt{8} \pi^{2}}\right)^{3} m_{H}^{6}\right]
$$

This implicitly gives the relation between the Higgs quartic couplings $\lambda=G_{F} m_{H}^{2} /\left(\sqrt{8} \pi^{2}\right)$ and $\lambda^{P S}=G_{F} M^{2} /\left(\sqrt{8} \pi^{2}\right)$, defined in the two schemes. $G_{F}=1.16637 \cdot 10^{-5} \mathrm{GeV}^{-2}$ is the Fermi constant. It is straightforward to use eq. 3 for translating the expansions of eqns. 2 in the pole renormalization scheme, and thus obtain the correction factors $K_{1}$ and $K_{2}$ in the pole scheme. Of course, the tree level widths are given by $\Gamma_{\text {tree }}^{O S}=a_{1} m_{H} \lambda$, and $\Gamma_{\text {tree }}^{P S}=a_{1} M \lambda^{P S}$.

We plot the correction factors $K_{1}$ and $K_{2}$ in the on-shell and the pole schemes in figs. 1 and 2, for several values of the Higgs mass. In order to compare the two renormalization schemes, one has to fix the relative values of the Higgs masses in the two schemes, in a way which would correspond in a certain sense to the same physical situation. This is an ambiguous choice. If we relate the two masses by eq. 3 , this is equivalent to fix the real part of the position of the pole of the Higgs propagator at the same place. This is the convention we used in figs. 1 and 2. Other relations are of course possible. For example, an equally justified choice is to compare the corrections 
for values of the coupling constants for which the Higgs width is the same. Another possibility is to use the position of the peak of a given process to relate the coupling constants of the two schemes. Obviously, this choice is process dependent.

The factors $K_{1}$ and $K_{2}$, which are plotted in figs. 1 and 2, can easily be included in a Monte Carlo simulation in order to take into account the NNLO radiative corrections of enhanced electroweak strength to the Higgs lineshape. One only needs to multiply the tree level amplitude by the factor corresponding to the desired renormalization scheme. One notes that our expressions are only valid around the Higgs peak. Far from the resonance there is no reason to perform the Dyson summation, and one needs the exact self-energy and vertex functions instead of the momentum expansion which we use.

The size of the NNLO correction, compared to the NLO correction, is smaller in the pole scheme than in the on-shell scheme for the $\mu^{+} \mu^{-} \rightarrow H \rightarrow Z Z$ and $\mu^{+} \mu^{-} \rightarrow H \rightarrow, t \bar{t}$ processes in the resonance region. This behaviour can be seen clearly in fig. 3 , where we plot the absolute value of the correction factors $K_{1}$ and $K_{2}$. It agrees with similar results concerning the position of the Higgs pole in the complex plane [1], and it may be the result of a certain nonperturbative effect in the Higgs sector of the standard model [1, 2].

We regard this behaviour as an indication that the perturbative expansion converges better in the pole scheme than in the on-shell scheme, making the pole scheme preferable for studying the phenomenology of heavy Higgs bosons. This argument was discussed in some detail in refs. [1, 4, 7, 9], and we will not repeat it here. It relies on the assumption that the behaviour of the first few terms in the perturbative expansion is indicative for the divergence pattern of the asymptotic series. Of course, it is difficult to establish unambiguously if that is indeed the case without explicit knowledge of higher order corrections. Some insight in the behavoiur of higher orders can be obtained indirectly by studying the scale dependence of the results within some class of renormalization schemes, like for instance $\overline{M S}$ [14, 15]. Considering the complexity of the existing two-loop calculations in the Higgs sector, there is little hope that such physical processes will become available at three-loop order in the near future. One only has an incomplete picture of the Higgs sector in the strong coupling zone, and all existing information should be kept in mind.

\section{Acknowledgements}

We are indebted to Scott Willenbrock, Jochum van der Bij, George Jikia and Boris Kastening for interesting discussions. The work of A. G. is supported by the Deutsche Forschungsgemeinschaft (DFG). 

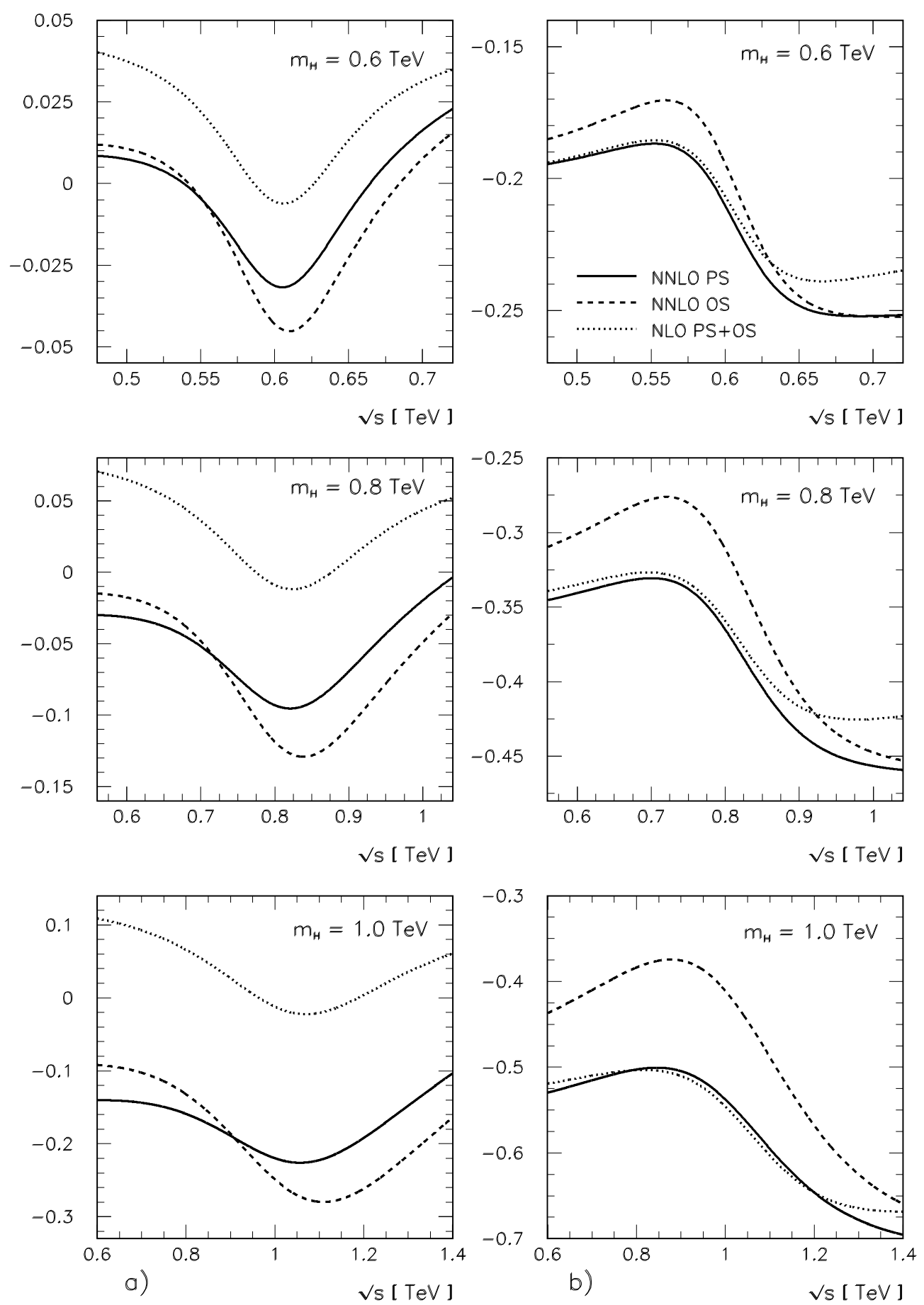

Figure 1: The real (a) and the imaginary part (b) of the radiative correction factor to the process $\mu^{+} \mu^{-} \rightarrow H \rightarrow Z Z$, for different values of the on-shell mass parameter $m_{H}$, which correspond to pole masses as defined in the text. We plot in this picture the value of $K_{1}-1$. 

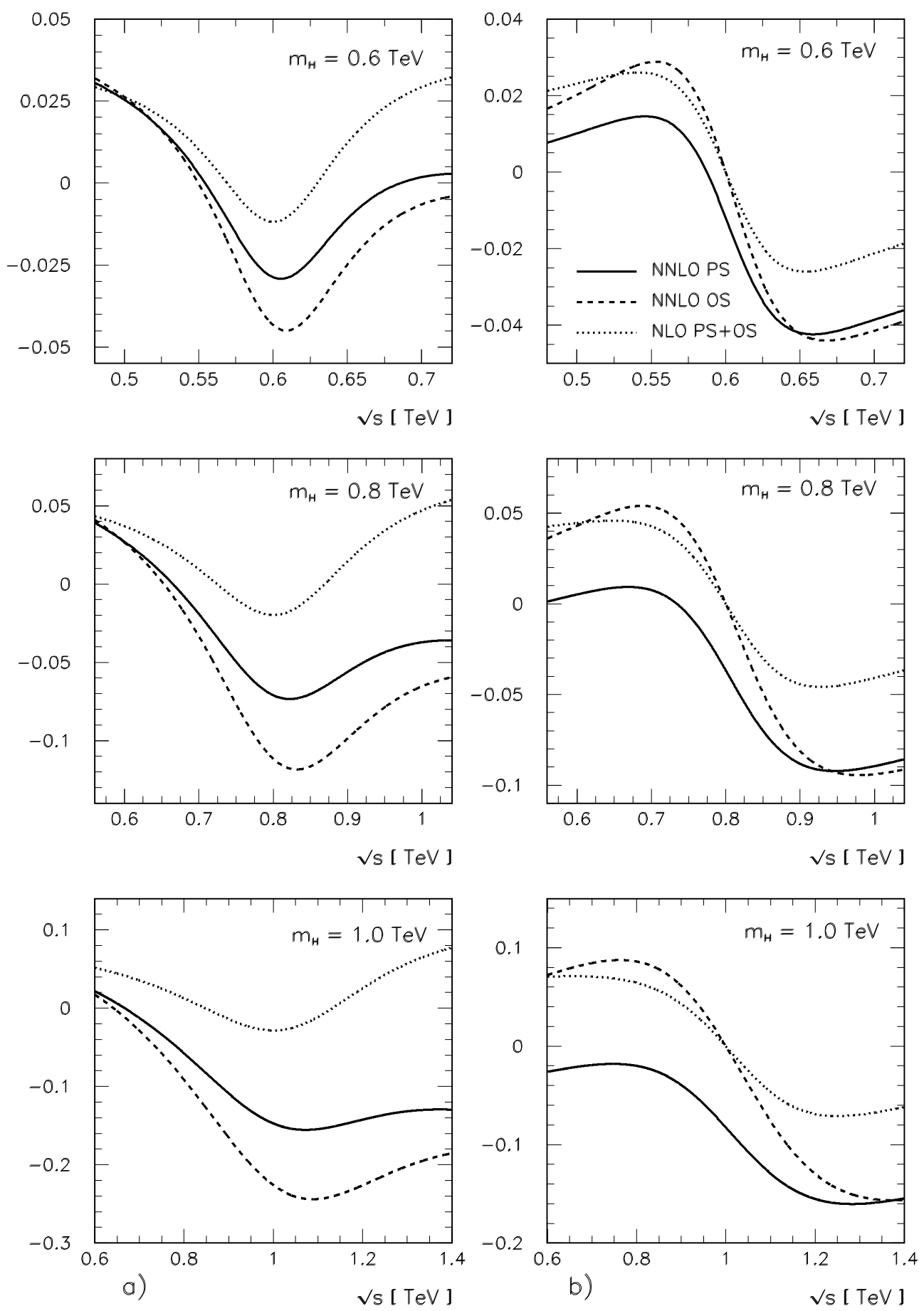

Figure 2: Same as fig. 1, but for the correction factor $K_{2}$ of the process $\mu^{+} \mu^{-} \rightarrow$ $H \rightarrow t \bar{t}$. 

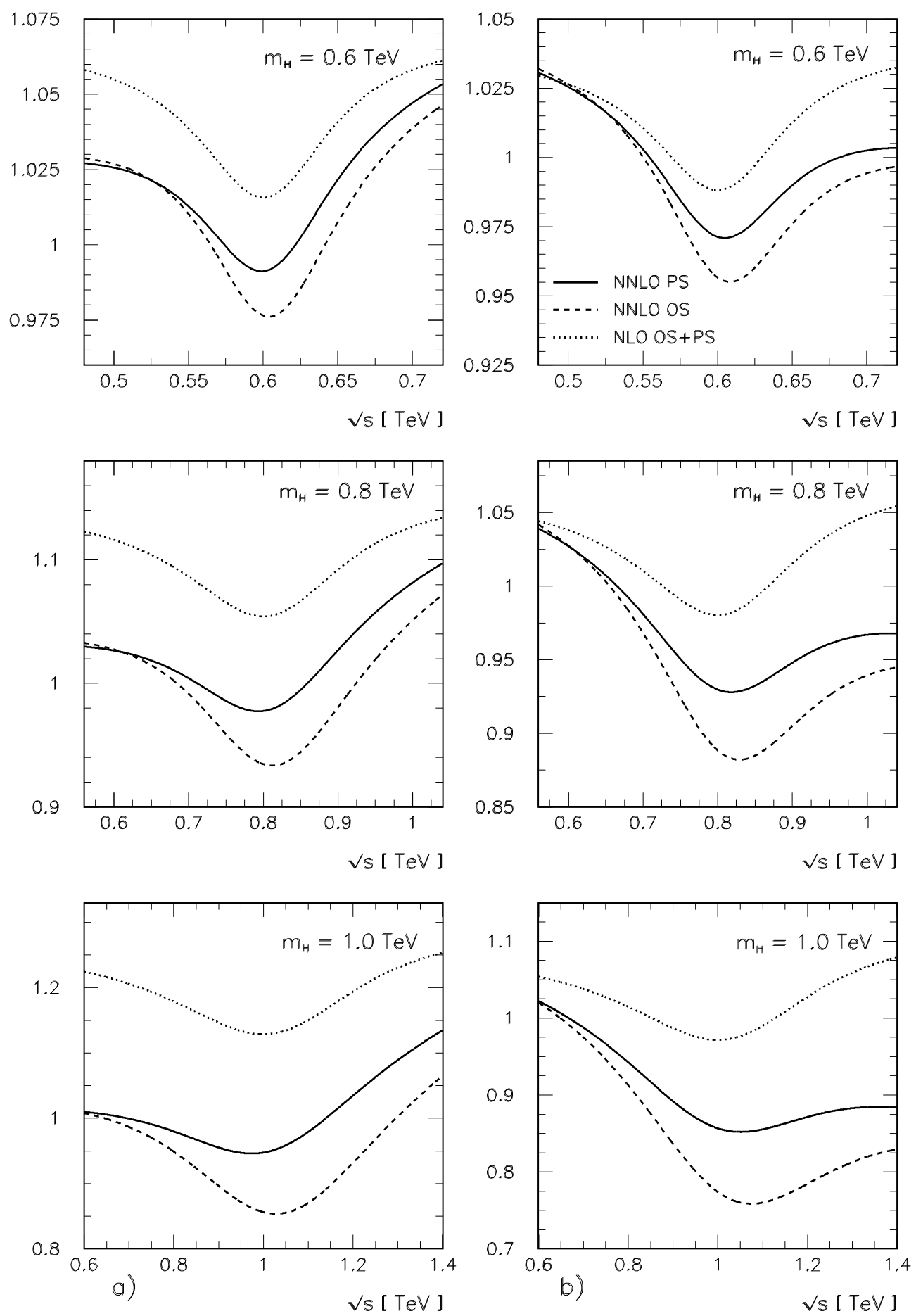

Figure 3: The absolute values of the radiative correction factors $K_{1}(a)$ and $K_{2}$ (b) for different values of the quartic coupling. We compare the pole and the on-shell schemes at values of the Higgs masses related as explained in the text. 


\section{References}

[1] A. Ghinculov, T. Binoth, Phys. Lett. B394 (1997) 139.

[2] W. Beenakker, G.J. van Oldenborgh, J. Hoogland, R. Kleiss, Phys. Lett. B376 (1996) 136.

[3] V. Barger, M.S. Berger, J.F. Gunion, T. Han, UCD-96-6 (1996), hepph/9602415.

[4] A. Ghinculov, Freiburg-THEP 96/14 (1996), hep-ph/9607455 and references therein.

[5] W.J. Marciano and S.S.D. Willenbrock, Phys. Rev. D37 (1988) 2509.

[6] S. Willenbrock and G. Valencia, Phys. Lett. B247 (1990) 341.

[7] A. Ghinculov, Nucl. Phys. B455 (1995) 21.

[8] A. Frink, B.A. Kniehl, D. Kreimer, K. Riesselmann, Phys. Rev. D54 (1996) 4548 .

[9] A. Ghinculov, Phys. Lett. B337 (1994) 137; (E) B346 (1995) 426.

[10] L. Durand, B.A. Kniehl and K. Riesselmann, Phys. Rev. D51 (1995) 500\%; Phys. Rev. Lett. 72 (1994) 2534; (E) Phys. Rev. Lett. 74 (1995) 1699.

[11] A. Ghinculov and J.J. van der Bij, Nucl. Phys. B482 (1996) 59.

[12] A. Ghinculov and J.J. van der Bij, Nucl. Phys. B436 (1995) 30.

[13] V. Borodulin and G. Jikia, Phys. Lett. B391 (1997) 434.

[14] U. Nierste, K. Riesselmann, Phys. Rev. D53 (1996) 6638.

[15] K. Riesselmann, S. Willenbrock, Phys. Rev. D55 (1997) 311. 